\title{
Osteoporosis for the practicing neurologist
}

\author{
Micol S. Rothman, MD \\ Sterling G. West, MD \\ Michael T. McDermott, MD
}

\section{Summary}

Osteoporosis is a common condition of impaired bone strength leading to fractures. A targeted history, physical exam, and blood work can help elucidate potentially reversible causes of low bone mass. In the neurology office, particular attention should be paid to the patient on glucocorticoids or antiepileptic medications, as these have distinct detrimental effects on bone. Patients can be risk-stratified by using the FRAX calculator, a tool that can help determine whether the patient is at sufficient risk of fracture to warrant pharmacologic therapy. Nonpharmacologic treatments such as calcium, vitamin D, and exercise should be discussed with the patient. The cornerstone of pharmacologic therapy has been treatment with bisphosphonates, but newer medications are available as well for the high-risk patient.

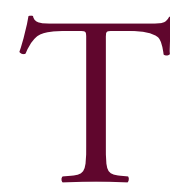

his article addresses the pathophysiology, diagnosis, and nonpharmacologic as well as pharmacologic treatment options for osteoporosis. Discussion is given to 2 particular areas of concern for neurologists-patients on glucocorticoids and antiepileptic medications.

\section{Clinical vignette}

A 62-year-old man is admitted to the hospital for a

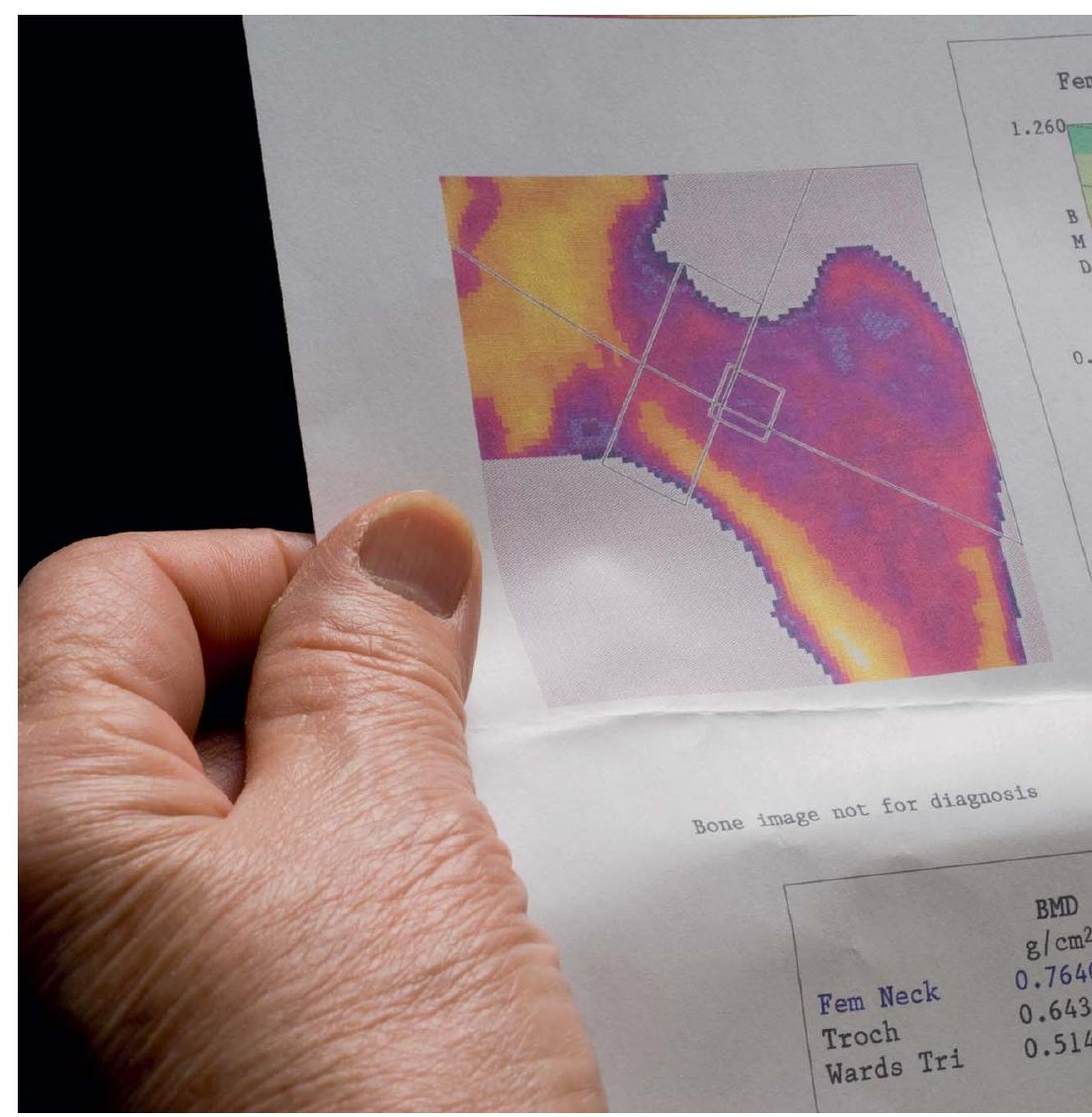

left hip fracture after slipping on ice in front of his house. His daughter notes he has a history of unsteady gait and long term use of medication for seizures. A bone density study reveals a $\mathrm{T}$-score of -2.7 at the right hip. What further workup and management is appropriate?

Department of Medicine-Endocrinology (MSR, MTM) and Department of Medicine-Rheumatology (SGW), University of Colorado School of Medicine, Denver, CO.

Funding information and disclosures are provided at the end of the article. Full disclosure form information provided by the authors is available with the full text of this article at Neurology.org/cp.

Correspondence to: Micol.Rothman@ucdenver.edu 
Table 1 Causes of low bone mass

$\begin{array}{ll}\text { Osteoporosis } & \text { Celiac disease } \\ \text { Osteomalacia } & \text { Inflammatory bowel disease } \\ \text { Osteogenesis imperfecta } & \text { Primary biliary cirrhosis } \\ \text { Hyperparathyroidism } & \text { Multiple myeloma } \\ \text { Hyperthyroidism } & \text { Rheumatoid arthritis } \\ \text { Hyperprolactinemia } & \text { Renal failure } \\ \text { Hypogonadism } & \text { Renal tubular acidosis } \\ \text { Cushing syndrome } & \text { Idiopathic hypercalciuria } \\ \text { Eating/exercise disorders } & \text { Systemic mastocytosis } \\ \text { High-risk medications } & \\ \text { a Well-established: glucocorticoids, excess thyroid hormone, antiepileptic drugs. Probable/possible: } \\ \text { thiazolidinediones, selective serotonin reuptake inhibitors, proton pump inhibitors. }\end{array}$

\section{Osteoporosis}

Epidemiology and pathophysiology Osteoporosis is defined as impaired bone strength that predisposes to the development of fragility fractures. ${ }^{1}$ It is a common condition, with more than 1.5 million fractures occurring in the United States each year. In normal bone remodeling, older, more fragile bone is replaced with newer, stronger bone. Osteoclasts are multinucleated giant cells that attach to bone surfaces where they secrete acid and proteolytic enzymes that dissolve underlying bone, leaving a resorption pit. Osteoblasts are then recruited to migrate in and secrete osteoid, a bone-specific collagen, which is subsequently mineralized with calcium and phosphate crystals (hydroxyapatite), refilling the resorption pit with new bone. Osteocytes are cells that reside in the bone matrix and function as mechanoreceptors, sensing areas of mechanical stress in bone and orchestrating the rate and sites of bone remodeling by sending signals to osteoclasts and osteoblasts. Osteoporosis most typically results from excessive resorption, but can also be related to low formation. ${ }^{2}$

Clinically, the diagnosis of osteoporosis can be made either by the presence of a fragility fracture, defined as a bone fracture that occurred with a fall from a standing height, or by bone density testing. Using bone densitometry criteria, osteoporosis is diagnosed when the lowest T-score (number of SDs the patient is below the average bone mineral density (BMD) for young normal adults) is $\leq-2.5$ and osteopenia is diagnosed when the lowest T-score is -1.0 to -2.4 . BMD is normal when T-scores at all sites are $>-1.0$. The most common fragility fractures are vertebral fractures, hip fractures, and wrist fractures. The most important risk factors for the development of fragility fractures are previous fragility fractures, low bone mass, age, and frequent falls.

\section{Evaluation of the patient}

A detailed history and physical examination should be undertaken, looking for potential reversible or secondary causes of osteoporosis. The history should note any factors that could have hindered optimal peak bone mass accrual or led to a precipitous decline in BMD. As outlined in table 1, these include a variety of conditions as well as osteoporosis risk factors themselves, such as prolonged amenorrhea in women, long-term tobacco and heavy alcohol use, inactivity, a lifetime of low intake of calcium and vitamin $\mathrm{D}$, and exposure to medications known to adversely affect BMD. Exam findings to note are any signs or symptoms of hyperthyroidism, bony deformities, bone pain, kyphosis or tenderness upon palpation of vertebrae, blue-tinged sclera, poor dentition, or abnormal gait. In most patients, a laboratory workup to evaluate for 


\section{Table 2 Evaluation for causes of secondary low bone mass}
1. Calcium
2. Creatinine (estimated glomerular filtration rate)
3. Alkaline phosphatase
4. $25(\mathrm{OH})$ vitamin $\mathrm{D}$
5. Total testosterone (men)
6. Thyroid-stimulating hormone (TSH)
7. 24-Hour urine calcium, sodium, creatinine

In select patients:

Celiac disease antibody testing

Serum and urine protein electrophorese

24-Hour urine cortisol or $1 \mathrm{mg}$ dexamethasone suppression

Parathyroid hormone

Serum tryptase

reversible or secondary causes of osteoporosis is appropriate. Experts differ on exactly what this should entail, but many agree that testing is appropriate (table 2).

\section{Nonpharmacologic management of osteoporosis}

Calcium, vitamin D, and weight-bearing exercise are important aspects of osteoporosis treatment. The 2010 Institute of Medicine (IOM) report advises 1,000 mg of calcium for all men aged 19-70 and women $19-50 .^{3}$ For women older than 50, they recommend 1,200 mg daily. ${ }^{3}$ A recent meta-analysis raised questions about potential harm from calcium, showing an increased risk of myocardial infarctions with calcium supplementation. ${ }^{4}$ Given these concerns, the IOM suggests not supplementing more than 2,000 $\mathrm{mg}$ of calcium daily. In our practice, we routinely ask patients about calcium intake, but only advise supplementation for those with inadequate dietary intake. A serving of milk, cheese, or yogurt provides about 300 $\mathrm{mg}$ of calcium; a more precise assessment can be done by having patients keep a food record of calcium intake.

Vitamin D is necessary for adequate absorption of calcium and phosphorus from the intestine. Fatty fish are rich in Vitamin $\mathrm{D}_{3}$, and several foods (milk, cereals) are fortified with either Vitamin $\mathrm{D}_{2}$ or $\mathrm{D}_{3}$; however, these dietary sources are often not sufficient. In addition, sunlight exposure is often brief or not suggested due to concerns about skin cancers, so oral vitamin $\mathrm{D}$ is the major source for most people. Endocrine Society guidelines recommend that adults between ages 19 and 50 years and lactating women take in at least 600 IU of vitamin D daily. ${ }^{5}$ For older adults, they suggest at least $600-800$ IU, but caution that in some groups this may not be adequate to maintain serum levels in the sufficient range. ${ }^{5}$ The IOM suggests $600 \mathrm{IU}$ for all age groups except for women over age 70, for whom they suggest 800 IU. ${ }^{3}$ Experts disagree on the target serum level of vitamin D, but many try to supplement to a $25(\mathrm{OH}) \mathrm{D}$ level at or above $30 \mathrm{ng} / \mathrm{dL}$, if vitamin $\mathrm{D}$ testing is available. This should be considered in the neurology patient who may be on medications that affect the metabolism of vitamin $\mathrm{D}$, as outlined further below.

Exercise should be done at least 3-5 times weekly and should consist of both aerobic and resistance work. This improves both bone and muscle strength and can reduce the risk of falling. Fall frequency and risks should be assessed at each visit, and measures should be instituted to correct or mitigate any identified fall risk factors, such the use of sedatives, visual impairment, musculoskeletal impairment, and obstacles to ambulation in the home environment. 
Table 3 Osteoporosis medications

\begin{tabular}{|llll|}
\hline $\begin{array}{l}\text { Medication } \\
\text { Bisphosphonates }\end{array}$ & Route & Dose & Frequency \\
\hline Alendronate & Oral & $10 \mathrm{mg}$ & Daily \\
\hline Risedronate & & $70 \mathrm{mg}$ & Weekly \\
\hline & Oral & $5 \mathrm{mg}$ & Daily \\
\hline Ibandronate & & $35 \mathrm{mg}$ & Weekly \\
\hline Zoledronic acid & & $150 \mathrm{mg}$ & Monthly \\
\hline Nonbisphosphonates & Oral & $150 \mathrm{mg}$ & Monthly \\
\hline Raloxifene & IV & $3 \mathrm{mg}$ & Every 3 months \\
\hline Calcitonin & IV & $5 \mathrm{mg}$ & Yearly \\
\hline Abbreviation: SC subcutaneous. & & & \\
\hline Estrogen & & $60 \mathrm{mg}$ & Daily \\
\hline Denosumab & Oral & $200 \mathrm{U}$ & Daily \\
\hline Anabolic agents & Nasal & $100 \mathrm{U}$ & Daily \\
\hline
\end{tabular}

\section{Pharmacologic therapy for the treatment of osteoporosis and osteopenia}

Determining which patients should receive pharmacologic osteoporosis therapy is the first issue. The National Osteoporosis Foundation guidelines advise medical treatment for anyone who has sustained a vertebral or hip fragility fracture or has a T-score $<-2.5$. For patients with osteopenia (T-score: -1.0 to -2.4 ) and no previous fragility fractures, the World Health Organization (WHO) Fracture Risk Assessment Tool (FRAX) (www.shef.ac.uk./FRAX) is a valuable adjunct to assist in making treatment decisions. Developed by the WHO and easily accessed online, this tool utilizes a weighted risk factor equation to estimate the 10-year probability of a fragility fracture. The National Osteoporosis Foundation recommends treatment for those whose 10 -year fracture risk calculates to $\geq 3 \%$ for hip fractures and $\geq 20 \%$ for other major osteoporosis fractures. Although a useful tool for risk stratification and discussion with patients, FRAX does have limitations. It does not take into account all known risk factors for low BMD, it does not weigh risks such as previous fractures (when we know the number of fractures affects future fractures) or dose of glucocorticoids, and it does not account for the presence of low spine BMD alone.

Pharmacologic agents, outlined in table 3 , are classified into 2 major mechanistic categories: antiresorptive medications and anabolic medications. Antiresorptive medications inhibit osteoclastic bone resorption and allow natural bone formation to continue for a variable period of time, resulting in a net gain of bone mass. Anabolic agents stimulate osteoblastic new bone formation, increasing bone mass by progressive bone accrual. ${ }^{6}$

Bisphosphonates are the most widely used antiresorptive medications for osteoporosis management. The US Food and Drug Administration (FDA)-approved bisphosphonates (alendronate, risedronate, ibandronate, and zoledronic acid) have all been documented to significantly increase BMD and reduce the risk of vertebral fracture. Alendronate, risedronate, and 


\section{Antiresorptive medications inhibit osteoclastic bone resorption and allow natural bone formation to continue for a variable period of time, resulting in a net gain of bone mass.}

zoledronic acid have also been shown to reduce the risk of hip fractures. ${ }^{7-13}$ The most common side effects with the oral bisphosphonates, seen in 10\%-30\% of patients, are heartburn and upper gastrointestinal pain. IV zoledronic acid, given once yearly, is particularly useful in patients with upper gastrointestinal side effects to oral agents or prior poor adherence to the prescribed oral dosing regimen. Oral bisphosphonates are approved for patients with a creatinine clearance of greater than $30 \mathrm{~mL} / \mathrm{min}$, while IV bisphosphonates should not be used when the clearance drops to less than $35 \mathrm{~mL} / \mathrm{min}$.

Concern about oversuppression of bone remodeling is an issue that has recently arisen in the context of prolonged use of potent antiresorptive medications like bisphosphonates. This has been fueled by the description of 2 rare conditions: antiresorptive-associated osteonecrosis of the jaw (ARONJ) and atypical femoral fractures, which are reported to occur at a rate of $1 / 10,000-1 / 100,000$. ONJ is defined as prolonged ( $\geq 8$ weeks) nonhealing bone and gums following invasive dental procedures (tooth extractions or implants). It has been reported most commonly in patients receiving frequent high doses of the IV bisphosphonates for treatment of cancer with bone lesions, but it has also been identified in some patients taking bisphosphonates for osteoporosis. ${ }^{14}$ There is no direct evidence that bisphosphonates were causative in these cases. A recent statement from the American Dental Association Council on Scientific Affairs advises dental examinations and oral care before and during bisphosphonate treatment, but cautions that there are no tests that predict which patients will develop ARONJ. ${ }^{15}$

There have been numerous case reports and case series describing atypical transverse femoral fractures in patients on bisphosphonates. Since all reported patients have had underlying low $\mathrm{BMD}$, it is not clear whether the risk for these fractures results from prolonged bisphosphonate use or the underlying osteoporosis. ${ }^{16}$ These fractures are deemed "atypical" because they occur in the subtrochanteric region of the femur and are often bilateral and not associated with trauma, which are not typical findings for osteoporotic hip fractures. A task force from the American Society for Bone and Mineral Research noted that these fractures are rare but do seem to increase in incidence with greater than 5 years of treatment with bisphosphonates. ${ }^{16}$

Given the above concerns, there are new recommendations from experts regarding duration of treatment with bisphosphonate therapy. ${ }^{17}$ Recent data suggest that those who are at the highest risk of fracture, as determined by $\mathrm{T}$-score and prior fracture, benefit the most from a longer duration of therapy. Experts advise re-evaluating patients' need for therapy after 3-5 years of treatment with bisphosphonates and suggest that those with T-scores still in the osteoporotic range or those with fractures may benefit from ongoing treatment, while those with T-scores above -2.0 who have not fractured are not likely to have continued benefit. ${ }^{17}$

The nonbisphosphonate antiresorptive medications currently available are estrogen replacement therapy, raloxifene, calcitonin, and the newest addition, denosumab. For the purpose of this review, we will focus on the more commonly prescribed of these treatments.

Denosumab is a monoclonal antibody that inhibits bone resorption by binding to RANK ligand, a major regulator of osteoclastic bone resorption. Denosumab is administered by subcutaneous injection every 6 months. It has been shown to significantly increase BMD and reduce the risk of vertebral, hip, and nonvertebral fractures. ${ }^{18}$ Contraindications include hypocalcemia and skin infections. Since denosumab works to inhibit bone resorption, the 


\section{Teriparatide should not be used in patients who have hypercalcemia or in any patient with an increased risk of developing bone cancer.}

same concerns exist for bone suppression with its use as with the bisphosphonates. Both ARONJ and atypical femoral fracture have been reported in patients receiving denosumab, although again, causality is not known. ${ }^{19}$

Teriparatide is the only anabolic bone agent currently approved in the United States. It is given by daily subcutaneous injection, and the anabolic action of this 34 amino acid fragment of intact parathyroid hormone (PTH) results from this pulsatile exposure, which is different from the continuous exposure seen with primary hyperparathyroidism. ${ }^{6}$ Teriparatide has been shown to increase BMD 2-3 times more than the antiresorptive medications and to reduce the risk of both vertebral and nonvertebral fractures. ${ }^{20,21}$ For glucocorticoid-induced osteoporosis (GIOP), teriparatide reduced vertebral fractures and raised spine BMD to a greater degree than oral alendronate. ${ }^{22}$ Teriparatide should not be used in patients who have hypercalcemia or in any patient with an increased risk of developing bone cancer. Osteosarcomas were reported in preclinical research in rats given 3-60 times the equivalent human dose of teriparatide, but an increased incidence of this tumor has not been demonstrated in humans. ${ }^{23}$ Teriparatide treatment is currently limited to a single 2-year course of therapy. Following this, treatment with an antiresorptive agent should be instituted to preserve the improvement in bone mass and strength achieved with teriparatide. ${ }^{24}$

\section{Special consideration for the neurology patient}

Glucocorticoids Glucocorticoids taken in supraphysiologic amounts cause bone loss by adversely affecting both bone formation and bone resorption. These medications directly suppress bone formation by inhibiting osteoblast activity through reduced osteoblastogenesis and increased osteoblast apoptosis. They also indirectly stimulate bone resorption through inhibition of osteoprotegerin, an endogenous antiresorptive cytokine, and centrally mediated suppression of gonadal steroid production. Steroids also appear to enhance apoptosis of osteocytes, which normally function as mechanoreceptors within the bone matrix. As a result of these multiple effects, bone is lost more rapidly with glucocorticoid therapy than in any other situation. ${ }^{25}$

The American College of Rheumatology and European guidelines on the prevention and treatment of GIOP are in general agreement. All patients should make lifestyle modifications including adequate intake of calcium and vitamin $\mathrm{D}$, smoking cessation, avoidance of excessive alcohol ( $>2$ drinks/day), and weight-bearing exercises. The decision to initiate a bone-active agent is based on risk stratification using the FRAX tool, lowest T-score value, and history of fragility fracture. Low risk is defined by a FRAX 10-year risk for a major osteoporotic fracture of $10 \%$ or less, medium risk as $10 \%-20 \%$, and high risk as over $20 \%$, T-score below -2.5 at any site, or a history of a previous fragility fracture. In clinical practice, any patient not on a bone-active agent with a T-score below -1.5 or a loss of $4 \%$ or more of their BMD after 1 year on glucocorticoids should be evaluated for more aggressive therapy.

Pharmacologic recommendations for postmenopausal women and men older than age 50 years either starting or currently on glucocorticoids with an anticipated duration of therapy of 3 or more months are as follows:

- Low-risk patients on prednisone (or equivalent) $\geq 7.5 \mathrm{mg} /$ day should start a bisphosphonate (alendronate, risedronate, or zoledronic acid) that is FDA-approved for GIOP.

- Medium-risk patients on any dose (including prednisone $<7.5 \mathrm{mg} /$ day) of glucocorticoids should start a bisphosphonate. 
- High-risk patients on any dose or duration (including $<3$ months) of glucocorticoids should start a bisphosphonate. Teriparatide is an option for high-risk patients who have the lowest T-scores (below -2.5 ) or a history of fragility fracture.

Premenopausal women and men younger than age 50 years who have had a previous fragility fracture and are on prednisone $\geq 5 \mathrm{mg}$ /day should receive a bisphosphonate regardless of their FRAX score or T-score. Zoledronic acid or teriparatide are the best options for the patients who are on higher doses ( $\geq 7.5 \mathrm{mg} /$ day), those with a longer duration ( $\geq 3$ months), and those with the lowest T-scores (below -2.5). Therapeutic guidelines for premenopausal women with childbearing potential who have had a previous fragility fracture recommend a bisphosphonate only if taking $\geq 7.5 \mathrm{mg} /$ day. Oral risedronate theoretically may be the safest in this circumstance due to the potential of less fetal toxicity should the patient become pregnant. Teriparatide is an alternative option.

Therapeutic guidelines for the prevention and treatment of GIOP in patients receiving intermittent pulse glucocorticoids without daily therapy are lacking. Patients receiving 4 or more monthly IV pulses ( $1 \mathrm{~g}$ methylprednisolone equivalent) or high-dose oral pulses (prednisone $\geq 60 \mathrm{mg}$ /day with taper over 2-4 weeks) within a 12-month period are at risk and should be treated based on risk stratification outlined above.

Antiepileptic therapy Epilepsy is a common neurologic condition, and antiepileptic drugs (AEDs) are widely prescribed for patients with seizures, as well as other conditions such as migraines, chronic pain, and, most recently, obesity. It is estimated that $20 \%-65 \%$ of patients taking AEDs have low bone density, ${ }^{26,27}$ and a recent cross-sectional study estimated that each year of AED use increased fracture risk by $4 \%-6 \% .{ }^{28}$ Despite these and other data, fewer than $30 \%$ of patients with epilepsy are aware of an increased risk for fracture or falls, ${ }^{28}$ and neurologists are often unaware of these associations as well. ${ }^{27}$ The classic thought has been that the enzyme-inducing AEDS have a more detrimental effect on BMD; however, the newer agents may have a negative effect as well.

There are several means by which AEDs can adversely affect bone density. The most often discussed is that enzyme-inducing medications (such as phenytoin, phenobarbital, and carbamazepine) can lead to increased catabolism of vitamin D. These low $25(\mathrm{OH})$ vitamin D levels cause secondary hyperparathyroidism as well as decreased calcium absorption and resistance to PTH.

More recent data have shown bone loss in subjects using non-enzyme-inducing AEDs as well, ${ }^{29}$ which may be due to adverse effects on bone formation and resorption. ${ }^{30}$ The newer agents such as topiramate, zonisamide, and acetazolamide may also have a negative effect on bone by inducing a metabolic acidosis. ${ }^{27}$ Studies have been conflicting as to the effect of levetiracetam as well as some of the other new agents, with some studies shower lower BMD and others showing no increased risk. ${ }^{31-33}$ The increased risk of falls in patients with seizures remains a risk for fracture ${ }^{34}$ and does not depend on type of agent.

The mechanism and magnitude of the risk of AEDs are still not well understood, and there are no specific guidelines on the treatment of low bone density for patients on AEDs. However, given the concerns for low BMD and fracture risk, we do recommend educating patients and their caregivers on the importance of calcium, vitamin D, and weight-bearing exercise. Particular attention should be paid to vitamin $\mathrm{D}$, given the concerns for excess catabolism and subsequent osteomalacia. Screening bone densities should be done in postmenopausal women over 65 as in the general population, but may also be helpful for younger patients and male patients, particularly if they have other risk factors for fracture or have had fractures in the past. Those who meet criteria for pharmacologic therapy, either by BMD or FRAX score, as discussed above, should be treated.

\section{Conclusions and recommendations}

The figure illustrates an approach we employ for the general management of patients presenting with new or untreated osteoporosis. All people interested in the prevention or treatment of 
Figure Osteoporosis management algorithm

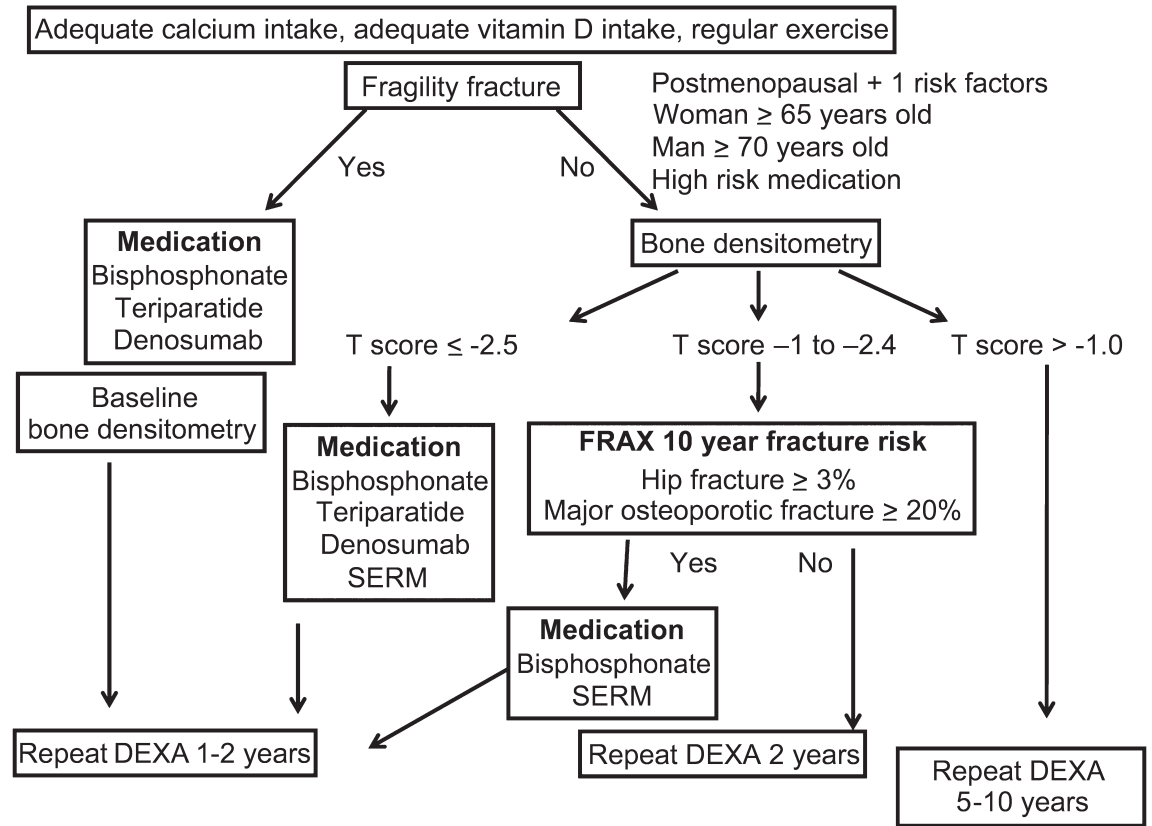

DEXA = dual-energy $\mathrm{x}$-ray absorptiometry; FRAX = Fracture Risk Assessment Tool; SERM = selective estrogenreceptor modulator.

osteoporosis should have adequate intake of calcium and vitamin D and should engage in regular exercise. Patients who have had a fragility fracture should have baseline bone densitometry testing and should be advised to start active medical therapy; based on fracture reduction data, the bisphosphonates and teriparatide are the best choices for patients with current or prior fractures. Bone densitometry testing should be ordered in postmenopausal women with one other risk factor, all women age 65 and older, all men age 70 and older, and those on high-risk medications. Treatment should be recommended for those with a T-score $\leq-2.5$; based on fracture reduction data, the bisphosphonates, teriparatide, and raloxifene are all good choices for these patients. The FRAX tool should be used to assist in treatment decisions for those with osteopenia; based on fracture reduction data, the bisphosphonates and raloxifene are good choices for these patients. For osteopenic patients with lower 10-year fracture risks and for patients with $\mathrm{T}$-scores in the normal range, preventive measures (calcium, vitamin $\mathrm{D}$, and exercise) should be recommended.

\section{Back to the patient}

Given the history of a low-impact fall with fracture, this patient has the diagnosis of osteoporosis regardless of what his bone density shows. However, bone density determination prior to starting therapy can help guide therapeutic choices and provide a baseline for future follow-up to determine effectiveness of therapy. He should have a secondary workup performed as outlined above and then appropriate counseling regarding calcium, vitamin D, weight-bearing exercise, and fall prevention. Given his long-term AED use, discussion around fall prevention and analysis of vitamin D values might prove particularly useful. He should be treated with approved pharmacologic therapy for osteoporosis after discussion of the risks and benefits with his provider.

\section{REFERENCES}

1. NIH Consensus Development Panel on Osteoporosis Prevention, Diagnosis, and Therapy. Osteoporosis prevention, diagnosis, and therapy. JAMA 2001;285:785-795. 
2. Raisz LG. Pathogenesis of osteoporosis: concepts, conflicts, and prospects. J Clin Invest 2005;115: 3318-3325.

3. Institute of Medicine. Dietary reference intakes for calcium and vitamin D. Available at: http://www.iom. edu/Reports/2010/Dietary-Reference-Intakes-for-calcium-and-vitamin-D.aspx. Accessed August 1, 2013.

4. Bolland MJ, Avenell A, Baron JA, et al. Effect of calcium supplements on risk of myocardial infarction and cardiovascular events: meta-analysis. BMJ 2010;341:c3691.

5. Holick MF, Binkley NC, Bischoff-Ferrari HA, et al. Evaluation, treatment, and prevention of vitamin D deficiency: an Endocrine Society clinical practice guideline. J Clin Endocrinol Metab 2011;96: 1911-1930.

6. Canalis E, Giustina A, Bilezikian JP. Mechanisms of anabolic therapies for osteoporosis. New Engl j med 2007;357:905-916.

7. Black DM, Schwartz AV, Ensrud KE, et al. Effects of continuing or stopping alendronate after 5 years of treatment: the Fracture Intervention Trial Long-term Extension (FLEX): a randomized trial. JAMA 2006;296:2927-2938.

8. Orwoll E, Ettinger M, Weiss S, et al. Alendronate for the treatment of osteoporosis in men. N Engl J Med 2000;343:604-610.

9. Harris ST, Watts NB, Genant HK, et al. Effects of risedronate treatment on vertebral and nonvertebral fractures in women with postmenopausal osteoporosis: a randomized controlled trial. Vertebral Efficacy With Risedronate Therapy (VERT) Study Group. JAMA 1999;282:1344-1352.

10. McClung MR, Geusens P, Miller PD, et al. Effect of risedronate on the risk of hip fracture in elderly women. Hip Intervention Program Study Group. N Engl J Med 2001;344:333-340.

11. Black DM, Delmas PD, Eastell R, et al. Once-yearly zoledronic acid for treatment of postmenopausal osteoporosis. N Engl J Med 2007;356:1809-1822.

12. Delmas PD, Recker RR, Chesnut CH III, et al. Daily and intermittent oral ibandronate normalize bone turnover and provide significant reduction in vertebral fracture risk: results from the BONE study. Osteoporos Int 2004;15:792-798.

13. Lyles KW, Colon-Emeric CS, Magaziner JS, et al. Zoledronic acid and clinical fractures and mortality after hip fracture. N Engl J Med 2007;357:1799-1809.

14. Khosla S, Burr D, Cauley J, et al. Bisphosphonate-associated osteonecrosis of the jaw: report of a task force of the American Society for Bone and Mineral Research. J Bone Miner Res 2007;22:1479-1491.

15. Hellstein JW, Adler RA, Edwards B, et al. Managing the care of patients receiving antiresorptive therapy for prevention and treatment of osteoporosis: executive summary of recommendations from the American Dental Association Council on Scientific Affairs. J Am Dent Assoc 2011;142:1243-1251.

16. Shane E, Burr D, Ebeling PR, et al. Atypical subtrochanteric and diaphyseal femoral fractures: report of a task force of the American Society for Bone and Mineral Research. J Bone Miner Res 2010;25: 2267-2294.

17. Black DM, Bauer DC, Schwartz AV, Cummings SR, Rosen CJ. Continuing bisphosphonate treatment for osteoporosis-for whom and for how long? N Engl J Med 2012;366:2051-2053.

18. Cummings SR, San Martin J, McClung MR, et al. Denosumab for prevention of fractures in postmenopausal women with osteoporosis. N Engl J Med 2009;361:756-765.

19. Paparodis R, Buehring B, Pelley E, Binkley N. A case of an unusual subtrochanteric fracture in a patient receiving denosumab. Endocr Pract 2013;19:e4-e8.

20. Neer RM, Arnaud CD, Zanchetta JR, et al. Effect of parathyroid hormone (1-34) on fractures and bone mineral density in postmenopausal women with osteoporosis. N Engl J Med 2001;344:1434-1441.

21. Orwoll ES, Scheele WH, Paul S, et al. The effect of teriparatide [human parathyroid hormone (1-34)] therapy on bone density in men with osteoporosis. J Bone Miner Res 2003;18:9-17.

22. Saag KG, Shane E, Boonen S, et al. Teriparatide or alendronate in glucocorticoid-induced osteoporosis. N Engl J Med 2007;357:2028-2039.

23. Andrews EB, Gilsenan AW, Midkiff K, et al. The US postmarketing surveillance study of adult osteosarcoma and teriparatide: study design and findings from the first 7 years. J Bone Miner Res 2012;27:2429-2437.

24. Rittmaster RS, Bolognese M, Ettinger MP, et al. Enhancement of bone mass in osteoporotic women with parathyroid hormone followed by alendronate. J Clin Endocrinol Metab 2000;85:2129-2134.

25. Weinstein RS. Clinical practice. Glucocorticoid-induced bone disease. N Engl J Med 2011;365:62-70.

26. Nakken KO, Tauboll E. Bone loss associated with use of antiepileptic drugs. Expert Opin Drug Saf 2010;9:561-571.

27. Meier C, Kraenzlin ME. Antiepileptics and bone health. Ther Adv Musculoskelet Dis 2011;3:235-243.

28. Shiek Ahmad B, Hill KD, O’Brien TJ, Gorelik A, Habib N, Wark JD. Falls and fractures in patients chronically treated with antiepileptic drugs. Neurology 2012;79:145-151.

29. Ensrud KE, Walczak TS, Blackwell TL, Ensrud ER, Barrett-Connor E, Orwoll ES. Antiepileptic drug use and rates of hip bone loss in older men: a prospective study. Neurology 2008;71:723-730. 
30. Verrotti A, Greco R, Latini G, Morgese G, Chiarelli F. Increased bone turnover in prepubertal, pubertal, and postpubertal patients receiving carbamazepine. Epilepsia 2002;43:1488-1492.

31. Beniczky SA, Viken J, Jensen LT, Andersen NB. Bone mineral density in adult patients treated with various antiepileptic drugs. Seizure 2012;21:471-472.

32. Koo DL, Joo EY, Kim D, Hong SB. Effects of levetiracetam as a monotherapy on bone mineral density and biochemical markers of bone metabolism in patients with epilepsy. Epilepsy Res 2013; 104:134-139.

33. Lee RH, Lyles KW, Sloane R, Colon-Emeric C. The association of newer anticonvulsant medications and bone mineral density. Endocr Pract Epub 2012 Sep 14.

34. Carbone LD, Johnson KC, Robbins J, et al. Antiepileptic drug use, falls, fractures, and BMD in postmenopausal women: findings from the women's health initiative (WHI). J Bone Miner Res 2010; 25:873-881.

\section{STUDY FUNDING}

No targeted funding reported.

\section{DISCLOSURES}

M.S. Rothman reports no disclosures. S.G. West serves on a scientific advisory board for Amgen and owns stock in Novartis. M.T. McDermott reports no disclosures. Full disclosure form information provided by the authors is available with the full text of this article at Neurology.org/cp.

\section{Related articles from other AAN physician and patient resources}

Neurology ${ }^{\circledR} \quad$ - www.neurology.org

Falls and fractures in patients chronically treated with antiepileptic drugs July 10, 2012;79:145-151.

Low bone mass in newly diagnosed multiple sclerosis and clinically isolated syndrome July 12, 2011;77:151-157.

\section{Continuum ${ }^{\circledR} \bullet$ www.ContinuumJournal.com}

Pregnancy, Epilepsy, and Women's Issues June 2013;697-714.

Neuroimaging of the Spine August 2008;188-207.

\section{Neurology Now ${ }^{\circledR} \bullet \quad$ www.neurologynow.com}

\section{Boning Up On MS}

January/February 2010;6:26-30. 